\title{
The nicotine-degrading enzyme NicA2 reduces nicotine levels in blood, nicotine distribution to brain, and nicotine discrimination and reinforcement in rats
}

\author{
Paul R. Pentel ${ }^{1}$, Michael D. Raleigh ${ }^{2 *}$, Mark G. LeSage ${ }^{2}$, Thomas Thisted ${ }^{3}$, Stephen Horrigann ${ }^{4}$, Zuzana Biesova ${ }^{3}$ \\ and Matthew W. Kalnik ${ }^{3}$
}

\begin{abstract}
Background: The bacterial nicotine-degrading enzyme NicA2 isolated from P. putida was studied to assess its potential use in the treatment of tobacco dependence.

Results: Rats were pretreated with varying i.v. doses of NicA2, followed by i.v. administration of nicotine at $0.03 \mathrm{mg} / \mathrm{kg}$. NicA2 had a rapid onset of action reducing blood and brain nicotine concentrations in a dose-related manner, with a rapid onset of action. A $5 \mathrm{mg} / \mathrm{kg}$ NicA2 dose reduced the nicotine concentration in blood by $>90 \%$ at $1 \mathrm{~min}$ after the nicotine dose, compared to controls. Brain nicotine concentrations were reduced by $55 \%$ at 1 min and $92 \%$ at 5 min post nicotine dose. To evaluate enzyme effects at a nicotine dosing rate equivalent to heavy smoking, rats pretreated with NicA2 at $10 \mathrm{mg} / \mathrm{kg}$ were administered 5 doses of nicotine $0.03 \mathrm{mg} / \mathrm{kg}$ i.v. over $40 \mathrm{~min}$. Nicotine levels in blood were below the assay detection limit 3 min after either the first or fifth nicotine dose, and nicotine levels in brain were reduced by 82 and $84 \%$, respectively, compared to controls. A $20 \mathrm{mg} / \mathrm{kg}$ NicA2 dose attenuated nicotine discrimination and produced extinction of nicotine self-administration (NSA) in most rats, or a compensatory increase in other rats, when administered prior to each daily NSA session. In rats showing compensation, increasing the NicA2 dose to $70 \mathrm{mg} / \mathrm{kg}$ resulted in extinction of NSA. An enzyme construct with a longer duration of action, via fusion with an albumin-binding domain, similarly reduced NSA in a $23 \mathrm{~h}$ nicotine access model at a dose of $70 \mathrm{mg} / \mathrm{kg}$.
\end{abstract}

Conclusions: These data extend knowledge of NicA2's effects on nicotine distribution to brain and its ability to attenuate addiction-relevant behaviors in rats and support its further investigation as a treatment for tobacco use disorder.

Keywords: Nicotine, Enzyme, Metabolism, Degradation, Addiction

\section{Background}

Nicotine is the principal addictive component of tobacco [1]. Available pharmacotherapies for the treatment of tobacco use disorder are aimed at modifying the effects of nicotine by either interacting with neuronal nicotinic cholinergic receptors (nicotine replacement therapy, varenicline) or the neurotransmitters mediating nicotine's effects in the brain (bupropion) [2]. These pharmacotherapies have been

\footnotetext{
* Correspondence: rale0011@umn.edu

${ }^{2}$ Minneapolis Medical Research Foundation, 701 Park Ave, Minneapolis, MN 55415, USA

Full list of author information is available at the end of the article
}

helpful for enhancing smoking cessation rates, but most quit attempts still end in failure [3]. New, more effective therapeutic strategies for modifying nicotine's effects on the brain are therefore of interest. One such approach is the use of nicotine vaccines to bind nicotine in blood and reduce its distribution to brain [4]. This pharmacokinetic strategy showed strong proof-of principle in animals but failed Phase III clinical trials when evaluated by intention-to-treat analysis (all subjects included) [5]. However, enhanced smoking cessation rates were observed in several nicotine vaccine studies in the subset of subjects with the highest antibody concentrations in blood [6,7]. This finding suggests that a 
pharmacokinetic approach with sufficient potency could have merit provided that the magnitude of effect on reducing brain nicotine levels is adequate.

An alternative pharmacokinetic strategy being investigated is a nicotine-degrading enzyme that can rapidly reduce nicotine concentrations in blood and nicotine delivery to brain $[8,9]$. It has been known for over 60 years that some bacteria living in proximity to tobacco plants can degrade nicotine [10]. The pathways responsible have been identified [11-13] and several of the enzymes involved have been cloned and expressed in purified form $[8,14]$. One such enzyme, NicA2 isolated from $P$. putida, can use nicotine as its sole carbon and nitrogen source [12]. It has been proposed [8] that NicA2 degrades nicotine through flavin-dependent catalytic oxidation to methylmyosmine, which is further hydrolyzed to pseudooxynicotine (PON). This pathway is distinct from that of nicotine metabolism in humans, where the conversion of nicotine to cotinine via CYP450 enzymes accounts for $80-90 \%$ of endogenous nicotine metabolism. The remainder is metabolized via minor pathways including conversion through 2'-hydroxynicotine to PON [15]. NicA2 mimics this minor pathway. Thus, smokers or users of tobacco products are already chronically exposed to PON and its metabolic intermediates. An initial study of PON safety in rats showed no adverse effects after 5 weeks of administration [8]. Among nicotine's metabolites in humans only nornicotine is known to share its addictive properties $[16,17]$. Degradation of nicotine to PON via NicA2 is therefore an attractive strategy for enhancing nicotine degradation and thereby reducing its effects.

Preliminary studies of NicA2 have characterized the in vitro properties of this enzyme pertinent to its potential therapeutic use [8]. NicA2 is a $52.5 \mathrm{kDa}$ protein which, when expressed in E. coli, is complexed with flavin adenine dinucleotide (FAD, a redox co-factor) as indicated by the recently published high-resolution crystal structure [18], and remains catalytically active after isolation without addition of any other components. NicA2 has high catalytic activity with $k_{\text {cat }}$ of $0.013 \mathrm{~s}^{-1}, K_{\mathrm{m}}$ of $0.092 \mu \mathrm{M}$, and $k_{\text {cat }} / K_{\mathrm{m}}=1.4 \times 10^{5} \mathrm{~s}^{-1} \cdot \mathrm{M}^{-1}\left(37^{\circ} \mathrm{C}\right)$, and it rapidly degrades nicotine in vitro at nicotine concentrations representative of serum concentrations in heavy smokers [8].

In a recent report [9], these initial findings have been extended showing that an $\mathrm{N}$-terminal 50-residue truncated form of NicA2 fused to an albumin binding domain (NicA2-J1) demonstrated a prolonged half-life. Pretreatment of rats with this enzyme substantially reduced nicotine distribution to brain. Pretreatment with the enzyme also reduced signs of withdrawal following a 1-week s.c. infusion of nicotine. To further explore the therapeutic potential of enzymatic degradation of nicotine NicA2 was administered to rats to establish its effects on nicotine concentrations in blood and brain over a range of NicA2 doses with both single and repeated doses of nicotine. In addition, we examined its effects on nicotine discrimination and self-administration, models of nicotine addiction widely used to evaluate pharmacotherapies for nicotine or tobacco use disorder.

\section{Results}

\section{In vitro characterization of NicA2-albumin-binding} domain fusion

Final purity was $>95 \%$ (visual estimate based on SDS-PAGE), with an endotoxin level of $<0.25 \mathrm{EU} / \mathrm{mg}$. The in vitro activity in the Amplex Red assay was indistinguishable between NicA2 and NicA2-ABD (Fig. 1).

\section{NicA2 selectivity in vitro}

NicA2 showed $40-49 \%$ activity, compared to nicotine, toward the nicotine metabolite nicotine-1'-N-oxide and the minor tobacco alkaloids nornicotine and anatabine, and no measurable activity toward the remainder of tested compounds (Table 1 ).

\section{Completeness of quenching of NicA2 activity by $\mathrm{MeOH}$ in vitro}

The ability of $\mathrm{MeOH}$ to quench NicA2 activity was tested in vitro by addition of NicA2, nicotine and $\mathrm{MeOH}$ in various sequences to blood or homogenized brain. Adding $\mathrm{MeOH}$ before mixing NicA2 and nicotine completely quenched NicA2 activity, yielding blood nicotine concentrations that did not differ from those of the BSA control (Fig. 2). In

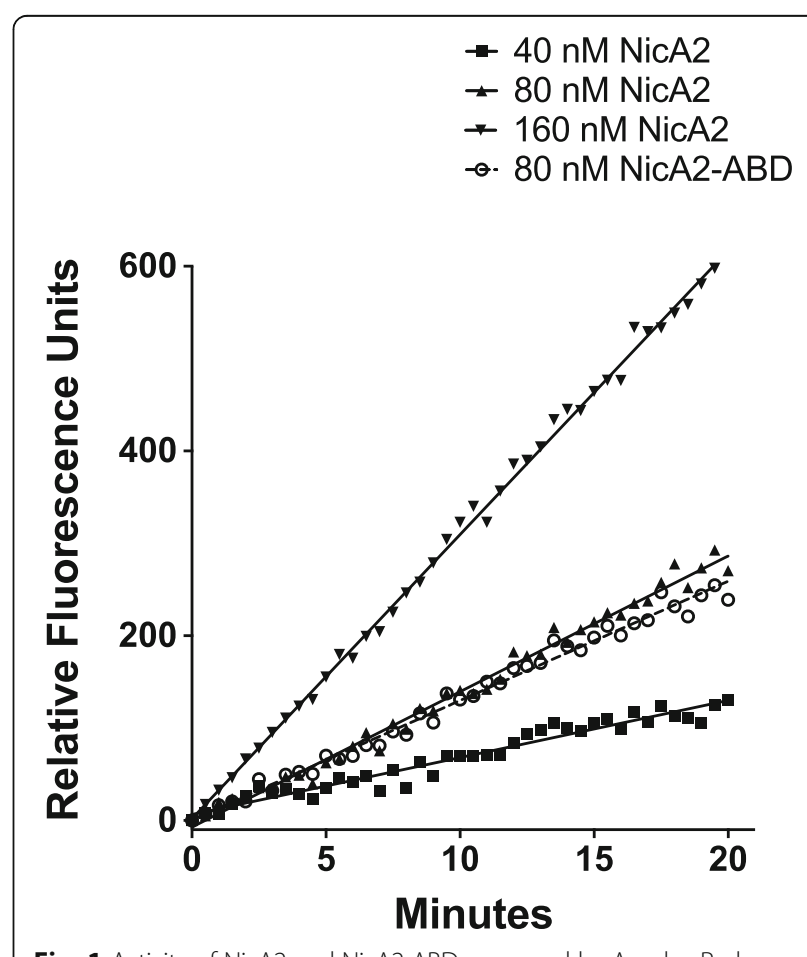

Fig. 1 Activity of NicA2 and NicA2-ABD measured by Amplex Red assay 
Table 1 NicA2 substrate specificity. Activity using the various compounds as substrates measured in an Amplex Red assay. Activities listed in percentages relative to that of nicotine. GABA; $\gamma$-amino-N-butyric acid, NAD; $\beta$-nicotinamide adenine dinucleotide

\begin{tabular}{ll}
\hline Compound & $\%$ Activity \\
\hline Nicotine & 100 \\
Anatabine & 49 \\
Nicotine-1'-N-oxide & 44 \\
Nornicotine & 40 \\
Anabasine & $<5$ \\
Myosmine & $<5$ \\
Norepinephrine & $<5$ \\
Dopamine & $<5$ \\
Serotonin & $<5$ \\
GABA & $<5$ \\
Choline & $<5$ \\
Acetylcholine & $<5$ \\
Nicotinamide & $<5$ \\
NAD & $<5$ \\
L-Glutamate & $<5$ \\
Cytisine & $<5$ \\
Mecamylamine & $<5$ \\
Varenicline & $<5$ \\
Bupropion & $<5$ \\
\hline
\end{tabular}

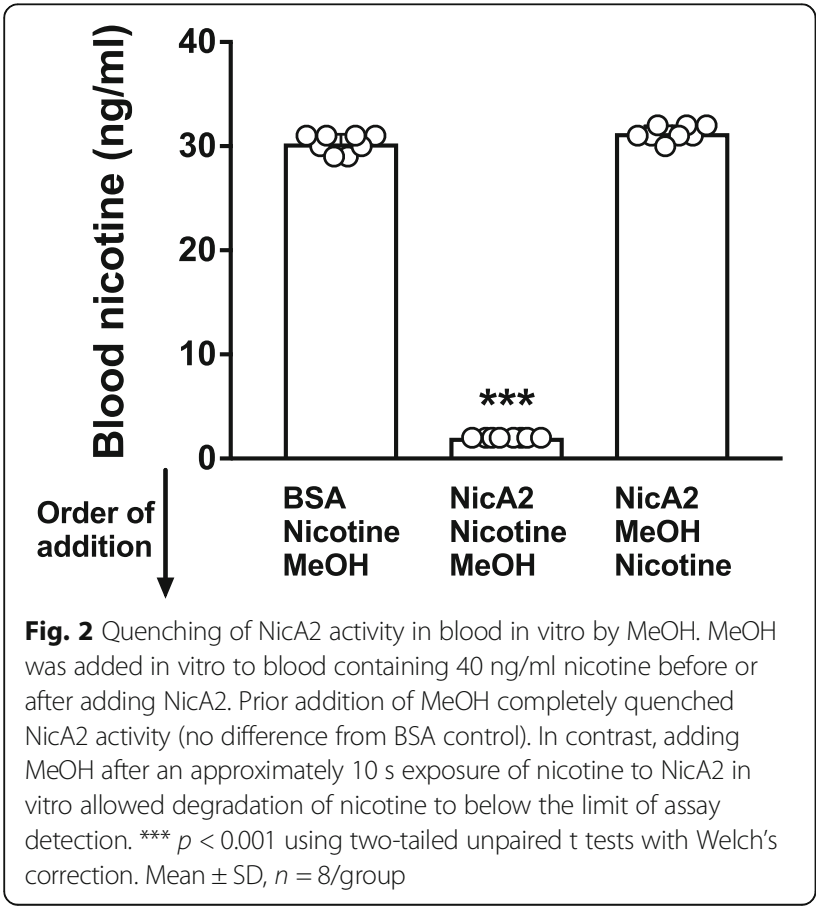

contrast, a delay of approximately $10 \mathrm{~s}$ in adding $\mathrm{MeOH}$ after mixing NicA2 and nicotine resulted in substantial degradation of nicotine, to below the $2 \mathrm{ng} / \mathrm{ml}$ limit of assay detection. Based on these results, in subsequent experiments all blood samples from animals were immediately placed in 4 volumes of methanol and vortexed prior to processing for measurement of nicotine concentrations. Similar results were obtained with brain homogenates and all brain samples in subsequent experiments were homogenized in $\mathrm{MeOH}$ (Fig. 3).

Comparison of NicA2 quenching from in vivo studies via immediate homogenization of brain in methanol v. Flash freezing brain prior to addition of $\mathrm{MeOH}$

There was no difference in measured nicotine concentrations in brain between the group immediately homogenized in $\mathrm{MeOH}$ and the group flash frozen and stored prior to addition of methanol $(86.2 \pm 3.4 \mathrm{ng} / \mathrm{g}$ v. $84.6 \pm$ $6.0 \mathrm{ng} / \mathrm{g}$, Mean $\pm \mathrm{SD}, p>0.4)$.

\section{Comparison of quenched v. Non-quenched brain from in vivo studies}

In control animals receiving BSA prior to nicotine, the measured nicotine concentration did not differ between those processed with or without homogenization in $\mathrm{MeOH}$ (Fig. 4). For rats pretreated with NicA2, brain nicotine levels were $27.5 \%$ lower (after a single nicotine dose, $p<0.05$ ) or $24.7 \%$ lower (after multiple nicotine doses, $p<0.05)$ when the $\mathrm{MeOH}$ step was omitted. Based on these data all brain tissue in subsequent experiments was rinsed in $\mathrm{MeOH}$ and immediately homogenized in 4 volumes of $\mathrm{MeOH}$ immediately after removal.

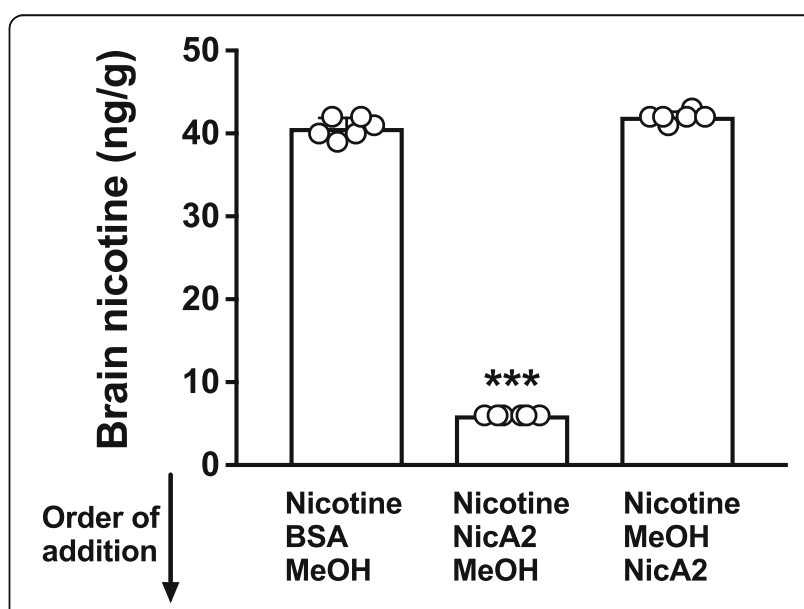

Fig. 3 Quenching of NicA2 activity in brain in vitro by $\mathrm{MeOH}$. Addition of $\mathrm{MeOH}$ to brain homogenate containing NicA2 prevented the degradation of subsequently added nicotine. However, a 10 s delay in adding $\mathrm{MeOH}$ to brain containing nicotine and NicA2 was sufficient to allow substantial degradation of nicotine. ${ }^{* * *} p<0.001$ using twotailed unpaired t tests with Welch's correction. Mean $\pm S D, n=6 /$ group 


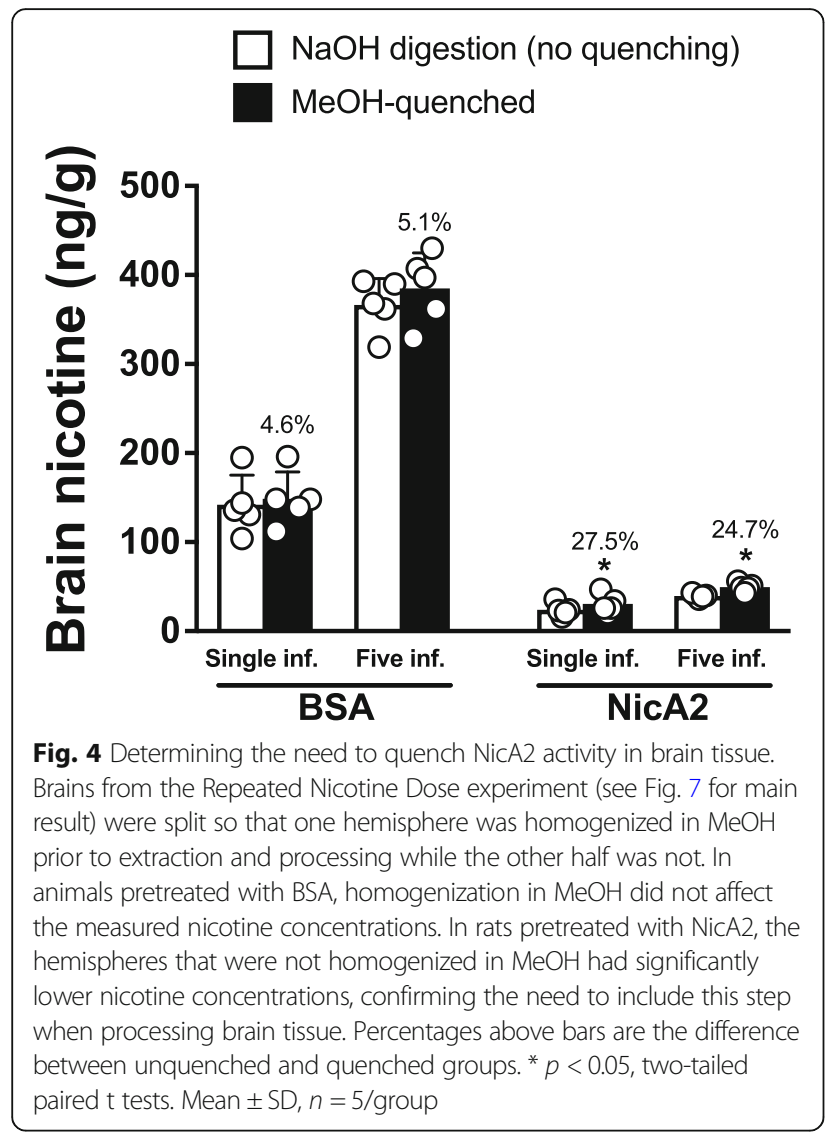

NicA2 and NicA2-ABD pharmacokinetic parameters

Enzyme concentration in serum was measured at intervals up to $24 \mathrm{~h}$ for NicA2 and 10 days for NicA2-ABD after IV dosing (Fig. 5). Parameters estimated by noncompartmental analysis of NicA2 concentrations include a serum half-life of $9.1 \pm 0.7 \mathrm{~h}$, clearance of $0.083 \pm 0.015 \mathrm{ml} / \mathrm{min} / \mathrm{kg}$, mean residence time of $11.6 \pm 1.9 \mathrm{~h}$, and steady state volume of distribution of $0.057 \pm 0.005 \mathrm{~L} / \mathrm{kg}$. For NicA2-ABD, parameters estimated by noncompartmental analysis include a serum half-life of $60.9 \pm 7.2 \mathrm{~h}$, clearance of $0.009 \pm$ $0.002 \mathrm{ml} / \mathrm{min} / \mathrm{kg}$, mean residence time of $80.7 \pm 6.1 \mathrm{~h}$, and steady state volume of distribution of $0.043 \pm 0.006 \mathrm{~L} / \mathrm{kg}$. All data are represented as mean \pm SD.

\section{NicA2 effects on blood and brain nicotine levels: Single nicotine dose}

The effects of a range of NicA2 doses on nicotine distribution to blood and brain, over periods of 1,3 or $5 \mathrm{~min}$, were analyzed (Fig. 6). NicA2 effects were dose and time dependent ( $p<0.0001$ by 2 -way ANOVA). NicA2 effects on blood or brain nicotine concentrations were substantial even at $1 \mathrm{~min}$ but were greater, particularly in brain, at $5 \mathrm{~min}$.

Blood nicotine levels were significantly lower than in controls at all sampling times in groups receiving NicA2 at doses $\geq 1.25 \mathrm{mg} / \mathrm{kg}$ and were reduced to < $2 \mathrm{ng} / \mathrm{ml}$ in all 64 rats receiving NicA2 doses of

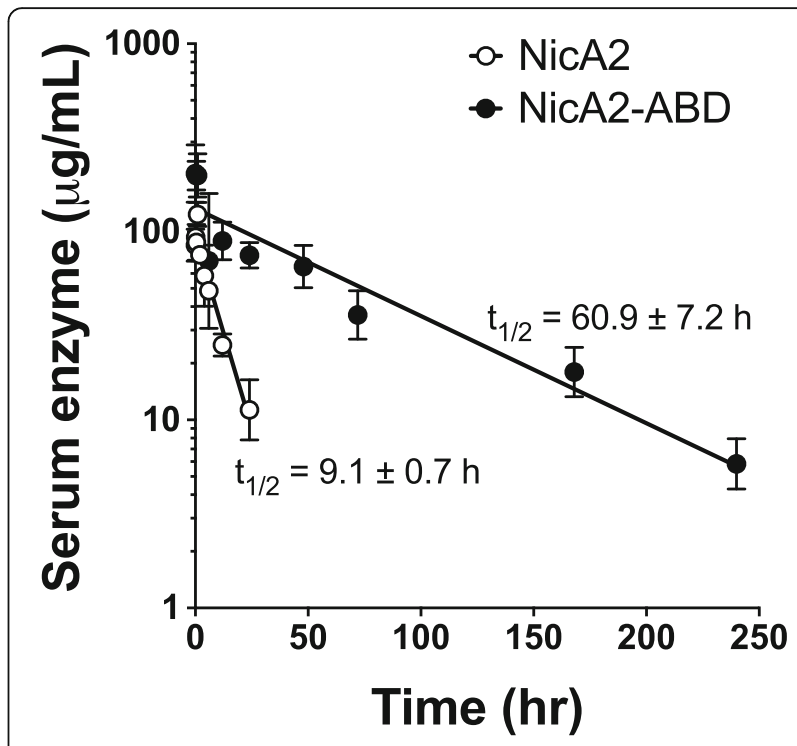

Fig. $5 \mathrm{NicA} 2$ and NicA2-ABD pharmacokinetics. Rats ( $n=3 /$ group) received either NicA2 or NicA2-ABD $5 \mathrm{mg} / \mathrm{kg}$ i.v. The terminal half-lives were determined by noncompartmental analysis. Data are the mean \pm SD of individual analyses

$\geq 5 \mathrm{mg} / \mathrm{kg}$. For rats receiving $\geq 5 \mathrm{mg} / \mathrm{kg} \mathrm{NicA} 2$ the blood nicotine level was reduced by $>90 \%$ at all sampling intervals compared to controls.

NicA2 efficacy in reducing brain nicotine levels was greater at 5 min than at earlier intervals. Brain nicotine levels were significantly lower than controls at $5 \mathrm{~min}$ in all groups receiving $\geq 0.31 \mathrm{mg} / \mathrm{kg} \mathrm{NicA} 2$, at $3 \mathrm{~min}$ in groups receiving $\geq 0.62 \mathrm{mg} / \mathrm{kg} \mathrm{NicA} 2$, and at $1 \mathrm{~min}$ in rats receiving $\geq 5 \mathrm{mg} / \mathrm{kg} \mathrm{NicA}$. Although $\geq 5 \mathrm{mg} / \mathrm{kg}$ NicA2 reduced brain nicotine levels by $95 \%$ at 3 and $5 \mathrm{~min}$, a higher dose of $20 \mathrm{mg} / \mathrm{kg}$ dose was needed to reduce brain nicotine levels to the same extent at $1 \mathrm{~min}$.

\section{NicA2 effects on blood and brain nicotine levels: Multiple nicotine doses}

Nicotine concentrations in blood and brain were significantly and substantially lower than controls in NicA2-treated rats receiving either a single nicotine dose or a series of 5 nicotine doses (Fig. 7). Blood nicotine concentrations were below the limit of assay detection for most rats receiving NicA2. Brain nicotine concentrations were reduced in rats receiving NicA 2 by $82 \%$ after the single nicotine dose and by $84 \%$ after the series of 5 nicotine doses, compared to their controls.

\section{Attenuation of nicotine discrimination by NicA2}

Baseline discrimination performance (left panel) and overall response rate (right panel) following the $0.4 \mathrm{mg} /$ $\mathrm{kg}$ nicotine training dose and the effects of NicA2 on 


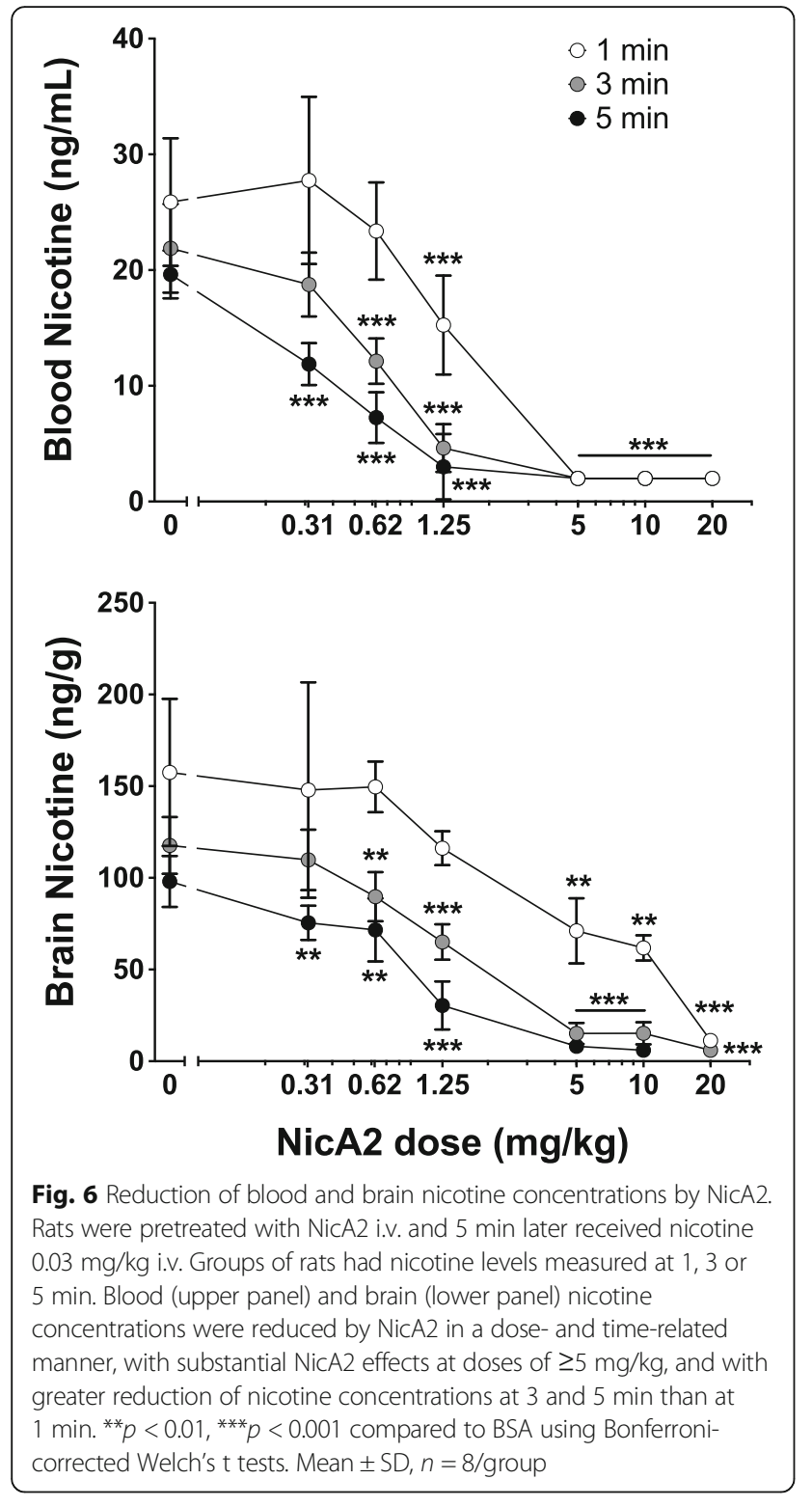

discrimination during substitution tests with $0.1 \mathrm{mg} / \mathrm{kg}$ nicotine are shown in Fig. 8. Substitution of the $0.1 \mathrm{mg} /$ $\mathrm{kg}$ nicotine dose following PBS vehicle pretreatment resulted in partial substitution $(72.3 \pm 15.15$ SD \% responding on the nicotine-appropriate lever) for the training dose. Following NicA2 pretreatment, percentage of responding on the nicotine-appropriate lever (\%NLR) was significantly reduced compared to vehicle (41.1 \pm 29.85 SD \%NLR, $t=3.36, p<0.05)$. There were no significant effects of NicA2 on response rate, although the higher response rate following saline versus $0.4 \mathrm{mg} / \mathrm{kg}$ nicotine and following $0.1 \mathrm{mg} / \mathrm{kg}$ nicotine + NicA2 versus $0.1 \mathrm{mg} / \mathrm{kg}$ nicotine + vehicle approached significance ( $p=0.06$ and 0.07 , respectively). The response rate data indicate that the decrease in \%NLR was not simply due to nonspecific suppression of motor activity.
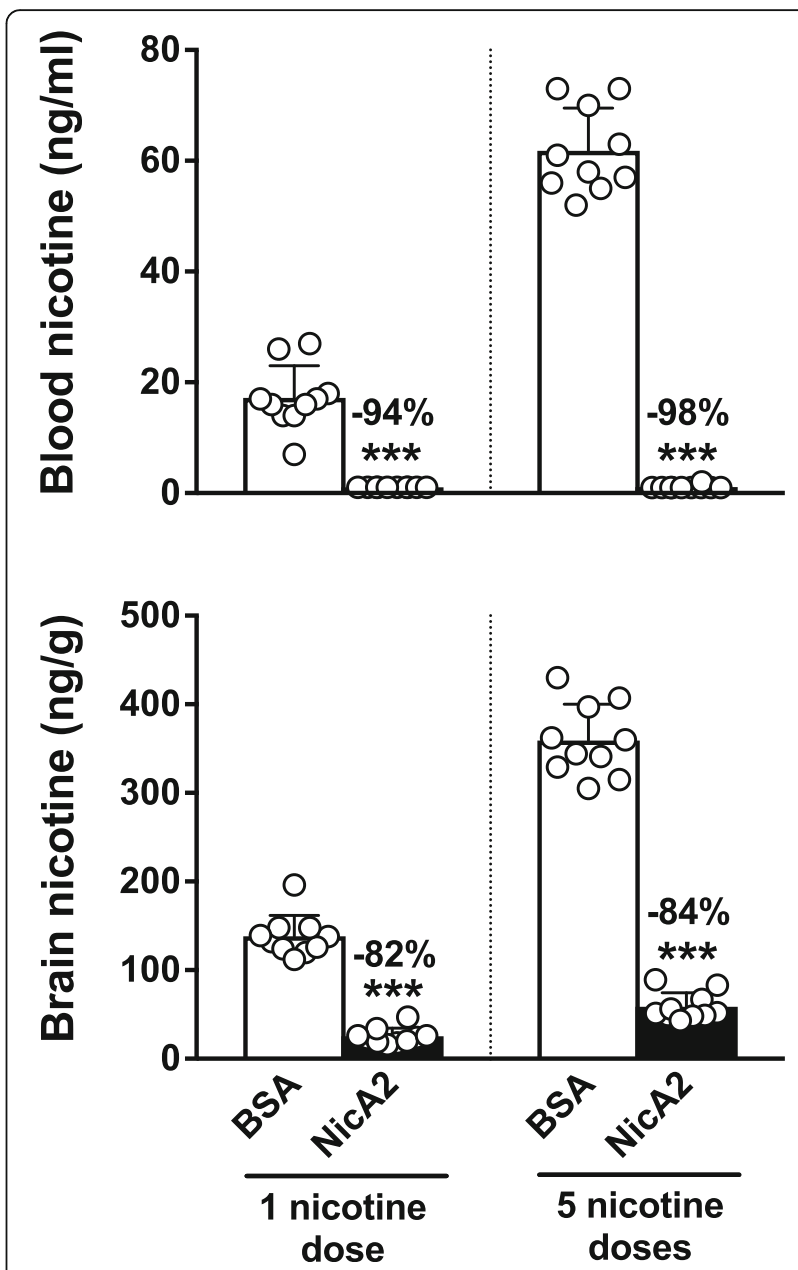

Fig. 7 Effects of NicA2 in rats receiving multiple nicotine doses. Rats were pretreated with either $10 \mathrm{mg} / \mathrm{kg} \mathrm{NicA} 2$ or BSA i.v. Five minutes later two groups received a single nicotine dose of $0.03 \mathrm{mg} / \mathrm{kg}$ i.v. and two groups received 5 nicotine doses at $10 \mathrm{~min}$ intervals. Blood and brain concentrations were measured 3 min after nicotine dosing. Numbers above bars are the percent reduction of nicotine concentrations compared to BSA control, in blood (upper panel) and brain (lower panel). ${ }^{* *} p<0.001$, two-tailed unpaired t tests with Welch's correction. Mean $\pm S D, n=10 /$ group

\section{Attenuation of nicotine self-administration by NicA2}

The purpose of this experiment was to examine the ability of NicA2 to block the reinforcing effects of nicotine in a self-administration model. Figure 9 shows nicotine self-administration (infusions earned) and sucrose-maintained responding (response rate) following vehicle pretreatment and NicA2 pretreatment over four consecutive test sessions. Pretreatment with PBS vehicle did not alter responding in either the nicotine or sucrose group (Panel A and B). Although there was no statistically significant effect of NicA2 when data from all eight rats were analyzed together, there was a clear dichotomy in the pattern of NSA between rats. Responding for nicotine decreased over the four NicA2 treatment sessions in five of eight rats 

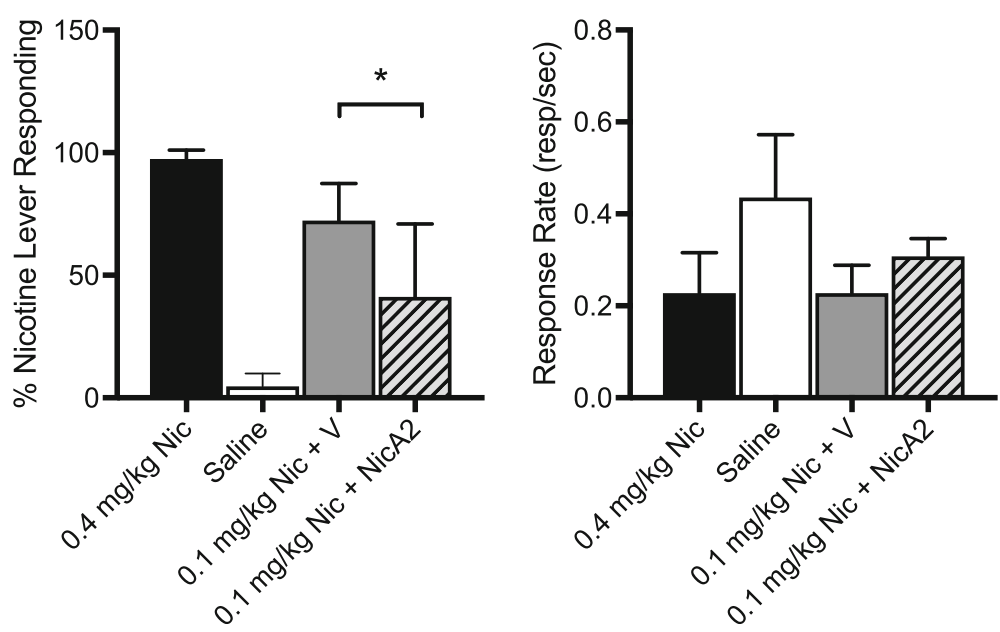

Fig. 8 Effect of NicA2 on nicotine discrimination. Each bar represents mean ( \pm SD) percent responding on the nicotine lever (left panel) or overall response rate (right panel) following administration of saline, the training dose $(0.4 \mathrm{mg} / \mathrm{kg})$, or the substitution dose $(0.1 \mathrm{mg} / \mathrm{kg})$ after PBS vehicle (V) or NicA2 pretreatment. * $p<0.05$, paired t test $(n=4)$

(Panel A; Decreasers). In these five rats NSA decreased by $65 \%$ by day four compared to vehicle. Four of these rats showed a brief increase in NSA on the first day of NicA2, similar to the extinction burst that occurs in some rats when saline is substituted for nicotine [19]. In contrast to the 5 Decreasers, 3 of the eight NSA rats exhibited a compensatory increase in NSA with the $20 \mathrm{mg} / \mathrm{kg}$ NicA2 dose

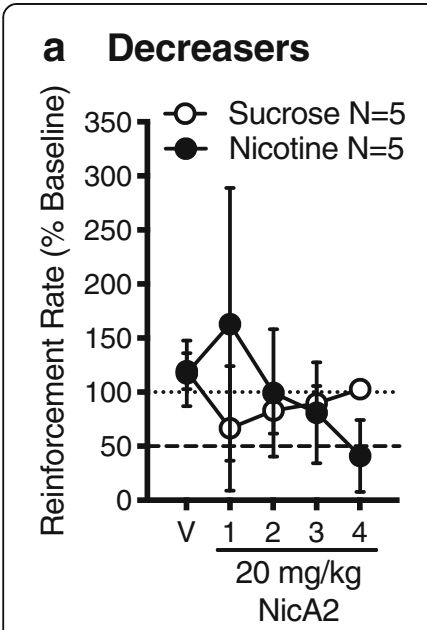

\section{b Compensators}

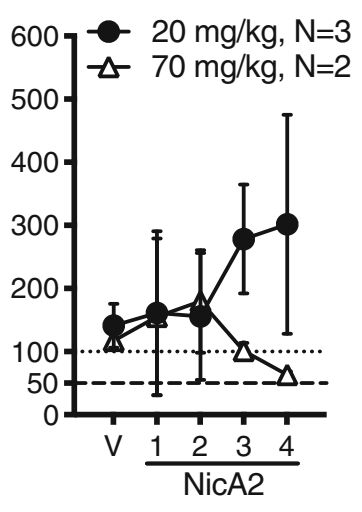

Fig. 9 NicA2 effects on nicotine or sucrose self-administration. Mean ( \pm SD) nicotine self-administration (infusion rate) and sucrose-maintained responding (pellet delivery rate) following pretreatment with PBS vehicle $(M)$ and NicA2 over four consecutive test sessions, expressed as a percentage of baseline. A total of 8 rats responding for nicotine were treated with NicA2. Of these, 5 showed a decrease in NSA (Panel $\mathbf{a}$; Decreasers) and 3 showed an increase in NSA (Panel b; Compensators). Two of these "compensators" were allowed to re-establish baseline NSA and were then treated with $70 \mathrm{mg} / \mathrm{kg} \mathrm{NicA2}$ (Panel b). Five additional rats responded for sucrose and received NicA2 (Panel a). The dotted horizontal line represents baseline. The dashed horizontal line represents the $50 \%$ reduction criterion for extinction
(Panel B; Compensators). However, increasing the NicA2 dose to $70 \mathrm{mg} / \mathrm{kg}$ in two of these animals decreased NSA by $37 \%$ by day four (Panel B). The third rat's catheter failed before the higher NicA2 dose could be tested. Pretreatment with NicA2 did not affect responding for sucrose compared to vehicle (Panel A). Two rats showed a significant decrease on day 1 of NicA2 treatment but returned to baseline across days 2 to 4 .

Figure 10 shows nicotine self-administration (infusions earned) following vehicle pretreatment and NicA2-ABD pretreatment $(70 \mathrm{mg} / \mathrm{kg}$ i.v. $)$ over six consecutive test sessions for four rats. PBS vehicle did not alter NSA, whereas NSA decreased over the six daily NicA2-ABD treatment sessions in all rats, with NSA significantly reduced by $74 \%$ by day six compared to vehicle $(t=6.99$, $p<0.01)$. One rat exhibited an extinction-like increase in NSA only on the first day of NicA2-ABD.

\section{Discussion}

The main findings from the present assessment of NicA2 activity in vivo are that pretreatment of rats with NicA2: 1) markedly reduced the early distribution of nicotine to brain when nicotine was administered as a single rapid i.v. bolus dose, 2) reduced nicotine distribution to brain when nicotine was administered as repeated i.v. doses at a $\mathrm{mg} / \mathrm{kg}$ rate comparable to heavy cigarette smoking, and 3) attenuated nicotine discrimination and nicotine reinforcement, effects that are predictive of the efficacy of smoking cessation medications. In addition, NicA2's elimination half-life of $9.1 \mathrm{~h}$ in rats was increased to $60.9 \mathrm{~h}$ by fusion with an albumin binding domain without impairing its catalytic activity. These data suggest that the enzymatic degradation of nicotine 


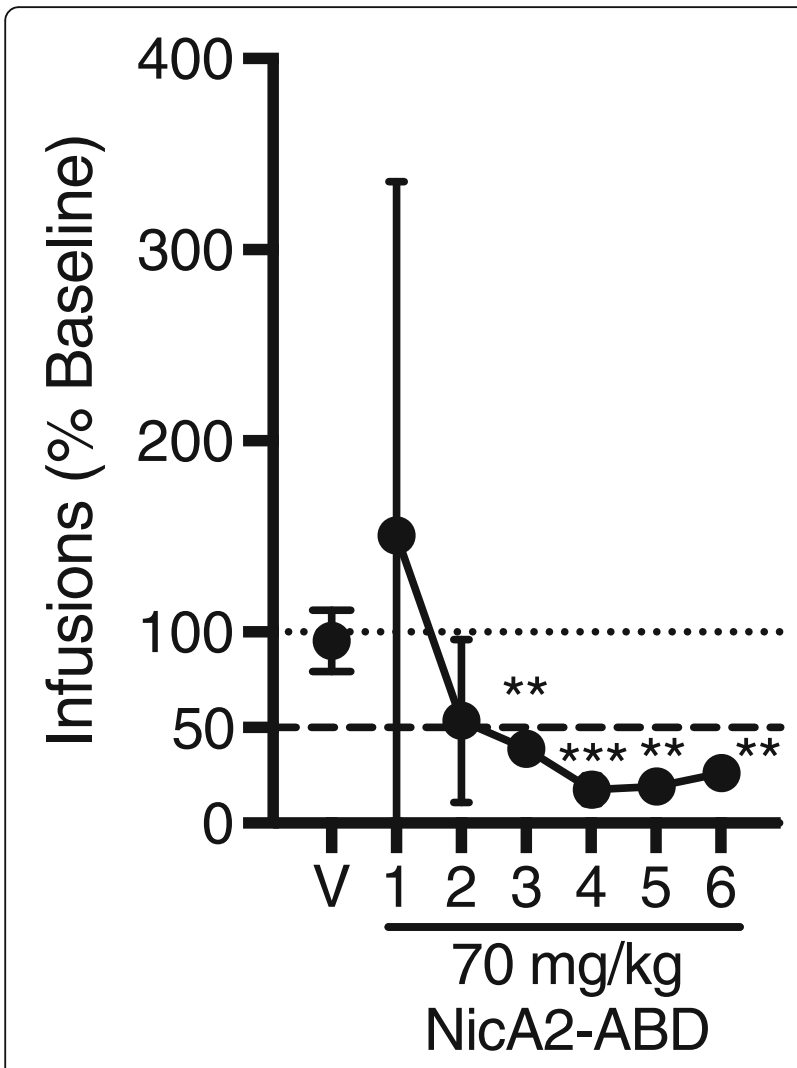

Fig. 10 Effects of NicA2-ABD on NSA during unlimited access to nicotine. Mean $( \pm S D)$ number of infusions during 23-h access following pretreatment with PBS vehicle $(V)$ and NicA2-ABD over six consecutive test sessions, expressed as a percentage of baseline. Each point represents the mean of four rats. The dotted horizontal line represents baseline. The dashed horizontal line represents the $50 \%$ reduction criterion for extinction. Different from $\mathrm{V}$, ${ }^{* *} p<0.01,{ }^{* * *} p<0.001$

via NicA2, with further optimization of its catalytic activity and attenuation of immunogenicity, is of interest as a potential smoking cessation medication.

An analogous approach has been explored with the use of cocaine-degrading enzymes for the treatment of cocaine use disorder or overdose. Experience with these enzymes is limited but helpful in assessing the therapeutic potential of NicA2. Two enzymes have shown considerable activity in clinical laboratory studies. A mutated bacterial enzyme RPB-8000, being developed as a treatment for cocaine overdose, was administered to human subjects at a dose of $200 \mathrm{mg} / \mathrm{kg} 1 \mathrm{~min}$ after an i.v. cocaine dose of $50 \mathrm{mg} / \mathrm{kg}$. This enzyme reduced the serum cocaine concentration by $90 \%$ within 2 min and reduced total exposure to cocaine (area under the time-concentration curve) by $95 \%$ [20]. An engineered variant of human butyryl cholinesterase (Alb-BChE; TV-1380), with increased catalytic activity and a longer serum elimination half-life than the wild-type, is also being developed as a potential therapeutic agent for cocaine use disorder. Pretreatment of human subjects with $100-300 \mathrm{mg} / \mathrm{kg}$ Alb-BChE reduced the peak plasma cocaine concentration and elimination half-life following an i.v. cocaine dose of $40 \mathrm{mg} / \mathrm{kg}$ by > $80 \%$, as well as its subjective effects [21]. These studies are consistent with preclinical data showing that a drug-degrading enzyme strategy can be effective in rapidly and substantially reducing cocaine plasma levels and attenuating its effects [22]. The high doses of these enzymes needed, however, have limited their clinical development.

The cocaine-degrading enzyme data are of particular interest because the single and daily doses of cocaine that are typically abused are more than an order of magnitude higher than those of nicotine delivered to cigarette smokers. Single cocaine doses of up to $40 \mathrm{mg}$ (approximately $0.6 \mathrm{mg} / \mathrm{kg}$ ) have been administered i.v. in clinical laboratory studies, with delivery of up to 7 doses of this size, or about $4 \mathrm{mg} / \mathrm{kg}$ over a $2.5 \mathrm{~h}$ session [23]. Self-reported doses of cocaine abused outside of a controlled setting may be considerably higher [24]. In contrast, one cigarette typically delivers about $1 \mathrm{mg}$ $(0.014 \mathrm{mg} / \mathrm{kg})$ of nicotine over $5-10 \mathrm{~min}$ as $5-10 \mathrm{di}-$ vided doses (puffs), about $3 \%$ the size of a typical laboratory cocaine dose [25]. A one pack per day smoker will receive about $20 \mathrm{mg}$ of nicotine over $12-16 \mathrm{~h}$, or $0.29 \mathrm{mg} / \mathrm{kg}$. This is about $7 \%$ of a potential cocaine laboratory session total dose. All else being equal, an enzyme-based drug lowering strategy should be more feasible for nicotine, since the amount of drug to be metabolized is considerably smaller and its rate of delivery considerably slower.

While the catalytic efficiency of NicA2 $\left(k_{\text {cat }} / K_{\mathrm{m}}\right.$ of $1.4 \times 10^{5} \mathrm{~s}^{-1} \mathrm{M}^{-1}$ ) [8] is lower than that of Alb-BChE $\left(k_{\text {cat }} / K_{\mathrm{m}}=2.3 \times 10^{7} \mathrm{~s}^{-1} \mathrm{M}^{-1}\right)$ [26] the NicA2 enzyme, unlike Alb-BChE has not yet undergone optimization to enhance its activity. More importantly, even at its current level of catalytic activity, NicA2 is highly effective in preventing nicotine from reaching brain in rats when nicotine is administered at single or multiple doses exceeding those received by heavy smokers. The effects of NicA2 were somewhat greater at $5 \mathrm{~min}$ after a nicotine dose than at $1 \mathrm{~min}$, but were nevertheless substantial at $1 \mathrm{~min}$. It is possible that the very rapid i.v. bolus delivery of nicotine in the current study, at doses higher than are delivered by a single puff of a cigarette, overwhelmed the catalytic capacity of the enzyme at $1 \mathrm{~min}$, and that NicA2 would prove more effective if nicotine were delivered to rats in smaller incremental doses, as in smokers. In support of this possibility, NicA2 blocked nicotine discrimination when the nicotine dose of $0.1 \mathrm{mg} / \mathrm{kg}$ was even larger than in the pharmacokinetic studies but was administered s.c. rather than i.v. and therefore absorbed more slowly.

Consistent with the effects of NicA2 on nicotine distribution to brain, NicA2 attenuated nicotine discrimination 
and decreased nicotine reinforcement in a nicotine self-administration model while it had no effect on responding for sucrose, i.e. NicA2's effects were specific for nicotine. Although the reinforcing effect of nicotine was attenuated in all rats, it was manifested in two distinct patterns. At the $20 \mathrm{mg} / \mathrm{kg}$ NicA2 dose most rats showed a moderate increase (extinction burst) in NSA, followed by a decrease to extinction-like levels by day 4 , while other rats showed only a compensatory increase in NSA, presumably to surmount decreased brain nicotine levels. However, this compensatory response was avoided by increasing the NicA2 dose. These data suggest that $20 \mathrm{mg} /$ $\mathrm{kg}$ is a near-threshold effective dose. The longer half-life of the NicA2-ABD fusion construct allowed it to be evaluated in a $23 \mathrm{~h} /$ day access NSA model, in which nicotine dosing more closely resembles the nicotine exposure of a smoker, and attenuation of nicotine reinforcement was confirmed.

Recently reported studies of a similar NicA2 albumin-binding domain enzyme fusion (NicA2-J1) [9] are consistent with and complementary to the current findings. When this enzyme was administered to rats during a 7-day nicotine infusion, it reduced signs of withdrawal following termination of the nicotine infusion compared to controls. Brain nicotine levels were below the limit of detection in the rats treated with NicA2-J1. This study supports the feasibility of extending NicA2's half-life to prolong its duration of effect.

The current study extends these findings by providing a) enzyme dose-response data over a range of time-frames, b) showing substantial enzyme effects on nicotine distribution to brain when nicotine is administered repeatedly, as i.v. boluses at doses comparable to heavy smoking c) showing effects of NicA2 on the key behavioral measures of nicotine discrimination and reinforcement, and d) confirming that extending NicA2's half-life via an albumin binding domain fusion does not affect its catalytic activity. It is noteworthy that NicA2 substantially blocked nicotine distribution to brain or its behavioral effects when nicotine was administered as large i.v. bolus doses. This method of nicotine delivery mimics the rapid uptake kinetics of nicotine from smoking [27].

Further support that a pharmacokinetic intervention could have therapeutic potential for smoking cessation comes from studies of nicotine vaccines and nicotine-specific monoclonal antibodies, both of which can reduce nicotine distribution to brain in animals and block addiction-relevant behaviors [4]. NicA2 is likely to be more potent than a nicotine vaccine or monoclonal antibody. In the current study, pretreatment of rats with NicA2 $10 \mathrm{mg} / \mathrm{kg}$ resulted in an $82 \%$ reduction in the distribution of a single nicotine dose to brain. In a previously reported study of the high affinity nicotine-specific monoclonal antibody Nic311 which used a similar protocol (nicotine $0.03 \mathrm{mg} / \mathrm{kg}$ i.v. and measurement of brain nicotine level $3 \mathrm{~min}$ after the dose), Nic311 80-160 mg/kg was required to produce a comparable reduction in nicotine distribution to brain [28]. In addition, NicA2 remained highly effective in reducing brain nicotine levels after 5 nicotine doses delivered over $40 \mathrm{~min}$ ( $>80 \%$ reduction in brain nicotine level compared to controls), while previous studies of a nicotine vaccine showed that its effects were considerably smaller after the same cumulative nicotine dose $(<30 \%$ reduction) [29]. Nicotine vaccines have shown signals of efficacy in clinical trials of smoking cessation with higher smoking cessation rates, albeit only in the subset of subjects that achieved the highest serum antibody concentrations $[6,7]$. If NicA2 proves more potent than nicotine-specific antibodies or vaccines in humans for reducing nicotine distribution to brain, as it appears to be in rats, it could also be more effective for enhancing smoking cessation rates.

A methodologic finding from this study is the importance of rapidly quenching blood or brain with $\mathrm{MeOH}$ to terminate NicA2 activity ex vivo. For blood, even a $10 \mathrm{~s}$ delay in doing so markedly reduced measured nicotine concentrations. Effects of quenching on brain nicotine levels were more limited, although still significant, presumably because little NicA2 enters brain tissue and only enzyme remaining in brain blood vessels needs to be quenched.

Several important hurdles must be addressed for NicA2 to be a useful therapeutic agent. Its serum elimination half-life needs to be lengthened to allow a suitable dosing frequency. The strategy of prolonging the enzyme's duration of action through fusion with an albumin-binding domain may be sufficient, as the serum half-life of albumin in humans is 3 weeks [30] but further study is needed to explore this option. A bacterial protein may be immunogenic and this potential must be minimized. Fortunately, the expected duration of treatment with enzyme needed for smoking cessation is relatively brief, typically 12 weeks based on experience with nicotine replacement therapy, bupropion and varenicline [2]. This relatively short expected duration of treatment should reduce the opportunity for development of anti-NicA2 antibodies. In addition, it would be desirable to further increase the catalytic activity of NicA2 both to enhance its effectiveness and to lower the required dose.

Components of tobacco or tobacco smoke, other than nicotine, are behaviorally active in various animal models $[17,31,32]$. Tobacco alkaloids including anabasine, anatabine and myosmine are present at much lower concentrations than nicotine and are also far less potent as reinforcers. Their contributions to tobacco use disorder or addiction, if any, are likely minimal $[32,33]$. Acetaldehyde is reinforcing in rodents [17] but at doses considerably higher than those delivered by smoking [34]. Tobacco pH may influence smoking behavior but does so by modifying 
nicotine absorption [1]. Monoamine inhibitors present in tobacco can modify nicotine's effects and enhance nicotine self-administration in rats but are not by themselves reinforcing [35]. In contrast, the evidence that the nicotine content of cigarettes drives tobacco use is abundant $[1,36]$. Reducing a smoker's exposure to nicotine using an enzyme such as NicA2, in order to promote smoking cessation, has a strong rationale.

Limitations of this study include the use of only one nicotine dose size in the pharmacokinetic experiments, and relatively small groups sizes in the behavioral studies. NicA2 had no obvious adverse effects in this study, aside from the decrease in sucrose-maintained responding in two rats on the first day of treatment but examining enzyme safety per se was not a specific goal. NicA2 showed high catalytic activity toward nicotine and, to a lesser extent, the nicotine metabolite nicotine- $\mathrm{N}$-oxide and the minor tobacco alkaloids nornicotine and anatabine, but did not metabolize any of the endogenous ligands or other compounds examined. A previous study showed no adverse effects of 5 weeks of dosing with pseudooxynicotine, the primary metabolite of nicotine through the action of NicA2. This is not surprising since pseudooxynicotine is a normally present minor metabolite of nicotine in humans [15]. Further studies of the safety of optimized versions of NicA2 will of course be needed.

\section{Conclusions}

NicA2 rapidly reduced blood and brain nicotine concentrations when administered to rats at single or multiple nicotine doses relevant to the nicotine intake of cigarette smokers. NicA2 also reduced the reinforcing potency of nicotine in a rat model of nicotine self-administration. These data establish NicA2 as a promising starting point for further optimization and development as a therapeutic agent and a novel treatment strategy for tobacco use disorder.

\section{Methods}

\section{NicA2 preparation and in vitro characterization}

NicA2 for in vitro studies was generated as described in [8]. For in vivo experiments, a similar expression construct was generated by cloning a synthetic gene encoding the same wildtype NicA2 amino acid sequence (GenBank: AEJ14620.1) [14] with an added C-terminal $\mathrm{His}_{6}$-tag (optimized for E. coli expression; GeneArt/Invitrogen) into $\mathrm{pET} 22 \mathrm{~b}(+)$, and was transformed into the $E$. coli expression strain BL21(DE3) (Agilent).

Purification of enzyme for in vivo testing added steps for endotoxin removal including $0.1 \%$ of the non-ionic surfactant octylphenol ethoxylate (Triton X-114; Sigma-Aldrich, St. Louis, MO) in the wash buffer during cobalt immobilized metal affinity chromatography purification (using
Talon resin; Clontech), followed by tangential flow filtration buffer exchange and an additional polishing step in the form of anion exchange chromatography using a $\mathrm{Q}$ Sepharose FF column (GE Life Sciences). Fractions containing NicA2 (by SDS-PAGE/Coomassie stain) were pooled, dialyzed into PBS pH 7.4 and concentrated. Concentration was determined by UV absorbance at $280 \mathrm{~nm}$ using the theoretically determined extinction coefficient $\mathrm{A}_{280}$ at $1 \mathrm{~g} / \mathrm{L}=1.313$ [37]. Endotoxin levels were determined using an Endosafe ${ }^{\circ}$ PTS $^{\mathrm{ma}}$ instrument (Charles River). Final purity was $>95 \%$ (visual estimate based on SDS-PAGE), with an endotoxin level of $0.12 \mathrm{EU} / \mathrm{mg}$.

Activity of purified protein was measured in vitro using the Amplex Red assay kit (Thermo). Based on NicA2's proposed mechanism, oxidation of nicotine results in the generation of $\mathrm{H}_{2} \mathrm{O}_{2}$ which is coupled to the conversion of the colorless Amplex Red reagent into its red-fluorescent product, resorufin by horseradish peroxidase [38]. Assays were performed as per the manufacturers protocol, including S-(-)-nicotine (Sigma) at a final assay concentration of $10 \mu \mathrm{M}$ in 96-well black half-area flat bottom plates (Corning). Fluorescence was detected in a SpectraMax M2 plate reader using excitation at $555 \mathrm{~nm}$, detection at $590 \mathrm{~nm}$, and employing a "Plate Blank" well to subtract the value derived from the no enzyme control for each point in the SoftMax ${ }^{\oplus}$ Pro data evaluation software package (Molecular Devices). Activities were expressed as the relative slopes of increase in fluorescence as a function of time. Development of fluorescence was dependent on the presence of nicotine, and the rate of fluorescence-development was proportional to the concentration of NicA2 in the range used.

\section{Substrate specificity of NicA2}

Substrate specificity of NicA2 was analyzed by the Amplex Red assay described above, using $10 \mu \mathrm{M}$ of test compound and $160 \mathrm{nM}$ NicA2 enzyme. Compounds tested were: (2'S)-nicotine-1'-N-oxide (Toronto Research Chemicals; TRC), ( \pm -nornicotine, nicotinamide, $\beta$-nicotinamide adenine dinucleotide (NAD), acetylcholine, choline, (-)-cotinine, varenicline, bupropion, (-)-cytisine, mecamylamine (TRC), dopamine, serotonin, $( \pm)$-norepinephrine, L-glutamate, $\gamma$-amino-N-butyric acid (GABA), (R,S)-anatabine (TRC), $(\mathrm{R}, \mathrm{S})$-anabasine (TRC), and myosmine (compounds obtained from Sigma-Aldrich unless otherwise stated). Activities were expressed relative to the activity found for S-(-)-nicotine run in parallel.

\section{Preparation of NicA2-albumin-binding domain fusion and in vitro characterization}

A gene fusion was prepared consisting of the NicA2 amino acid sequence mentioned above fused at its C-terminus to a $5 \mathrm{kDa}$ albumin binding domain (ABD035, which binds albumin with high affinity across rodents, 
non-human primates and humans [39]) via a flexible Gly4Ser linker followed by a C-terminal His -tag (gene optimized for E. coli expression; GeneArt/Invitrogen). This construct was cloned into $\mathrm{pET} 22 \mathrm{~b}(+)$ and transformed into the E. coli expression strain BL21(DE3) (Agilent). Expression and purification was carried out as described above.

\section{Nicotine assay and quenching of NicA2 activity}

Nicotine concentrations in blood or brain were measured using gas chromatography with nitrogen phosphorus detection $[40,41]$. Concentrations that were below the limit of quantitation for the assay were considered to be at the limit of $2 \mathrm{ng} / \mathrm{ml}$ for purposes of analysis. For this assay blood undergoes solvent extraction, while brain is first digested in $\mathrm{NaOH}$ before extraction. Because residual NicA2 in samples could continue to degrade nicotine ex vivo, blood samples were quenched immediately upon collection by drawing blood into a tube and transferring $0.5 \mathrm{ml}$ into 4 volumes of methanol and immediately vortexing.

Completeness of quenching of NicA2 activity in blood by $\mathrm{MeOH}$ was assessed in vitro by comparing samples prepared by adding the following to $0.5 \mathrm{ml}$ blood, in this order: a) BSA $100 \mu \mathrm{g} / \mathrm{ml} \rightarrow$ nicotine $40 \mathrm{ng} / \mathrm{ml} \rightarrow 4$ volumes of methanol; b) NicA2 $100 \mu \mathrm{g} / \mathrm{ml}$ (the approximate blood concentration of NicA2 following a $10 \mathrm{mg} /$ $\mathrm{kg}$ i.v. dose) $\rightarrow$ nicotine $40 \mathrm{ng} / \mathrm{ml} \rightarrow 4$ volumes of methanol; c) NicA2 $100 \mu \mathrm{g} / \mathrm{ml} \rightarrow 4$ volumes of methanol $\rightarrow$ nicotine $40 \mathrm{ng} / \mathrm{ml}$. Tubes were vortexed for $5 \mathrm{~s}$ at each step. Samples were stored at $-20{ }^{\circ} \mathrm{C}$ until assayed for nicotine levels. A similar protocol was performed using brain homogenate containing $40 \mathrm{ng} / \mathrm{ml}$ nicotine in place of blood, to determine completeness of quenching of brain samples by $\mathrm{MeOH}$ in vitro. Nicotine concentrations were compared using two-tailed unpaired $t$ tests with Welch's correction and adjusted for multiple comparisons $(\alpha=0.025)$.

For in vivo studies blood was obtained either through an indwelling venous catheter or as trunk blood after decapitation, and NicA2 activity was quenched as above. Brain was rapidly removed after decapitation, rinsed in methanol, and immediately homogenized with 4 volumes of methanol. At this point brain was stored at $20{ }^{\circ} \mathrm{C}$ with no loss of nicotine concentration. Alternatively, to facilitate storage and shipping of samples, brain could be rinsed, flash frozen in liquid nitrogen and stored at $-80{ }^{\circ} \mathrm{C}$ until assayed. When ready for assay, the frozen sample was placed in 4 volumes of methanol and processed as above. The adequacy of flash-freezing brain prior to addition of methanol was evaluated by pretreating 5 rats with $1.25 \mathrm{mg} / \mathrm{kg}$ NicA2 and administering nicotine $0.03 \mathrm{mg} / \mathrm{kg} 5 \mathrm{~min}$ later (equivalent to two cigarettes in a human). Brains were collected $3 \mathrm{~min}$ after nicotine administration. Brains were rinsed in methanol and one hemisphere of each brain was immediately homogenized in 4 volumes of methanol while the other half was flash frozen and stored at $-80{ }^{\circ} \mathrm{C}$ until assayed. Groups were compared with a two-tailed paired $\mathrm{t}$ test.

Because it was expected that very little NicA2 would cross the blood-brain barrier owing to its molecular weight of $52.5 \mathrm{kDa}$, it was initially unclear whether brain, after rinsing in methanol, required further quenching by methanol. This question was addressed by dividing samples obtained as part of the repeated nicotine dose pharmacokinetic experiment described below. In this experiment brain was obtained from rats that had been pretreated with NicA2 and then received either 1 or 5 doses of nicotine. Brains were first rinsed in $\mathrm{MeOH}$ and then split so that one hemisphere was processed only by rinsing the whole brain in methanol and the other hemisphere was processed by immediately placing it in 4 volumes of methanol and homogenizing. Groups were compared using two-tailed paired $t$ tests.

\section{Estimation of NicA2 and NicA2-ABD pharmacokinetic parameters}

Female Sprague Dawley rats weighing 225-250 g were obtained with a jugular venous catheter in place (Charles River). The choice of male or female rats in this and subsequent experiments was depending upon their availability and desired weight range at the time of each experiment. An additional goal was to test efficacy of NicA2 in both male and female rats. Three rats received $5 \mathrm{mg} / \mathrm{kg}$ His-tagged NicA2 via the tail vein. Blood $(0.2 \mathrm{ml})$ was collected into serum separator tubes via the jugular catheter at pre-dose and over a $5 \mathrm{~min}-24 \mathrm{~h}$ period for NicA2 or 5 min-10 days for NicA2-ABD, and serum was isolated and stored at $-20{ }^{\circ} \mathrm{C}$ until analysis. Assay of NicA2 or NicA2-ABD concentrations in serum samples took advantage of the C-terminal His-tag. MaxiSorp ELISA plates (Nunc) were coated overnight with anti-His tag antibody (R\&D Systems). Plates were blocked with $1 \%$ non-fat dry milk (NFDM) in PBS for approximately $1 \mathrm{~h}$. Dilutions of NicA2 or NicA2-ABD standards (for the latter a pre-incubation step in rat serum was conducted so the standard curve would accurately represent the NicA2-ABD:albumin complex detection in the actual samples) and serum samples in $1 \%$ NFDM in PBS $+0.1 \%$ Tween-20 were added to the plates and incubated for $2 \mathrm{~h}$ at room temperature. After washing away unbound substances (all wash steps performed in PBS $+0.1 \%$ Tween-20), rabbit anti-NicA2 polyclonal primary detection antibody (custom reagent generated by Noble Life Sciences) was added to the wells for a $1 \mathrm{~h}$ incubation. A wash step was followed by addition of horseradish peroxidase-conjugated goat anti-rabbit IgG 
(Fc) (KPL International). Plates were washed, and the remaining binding complex was detected with TMB substrate (3,3',5,5' -tetramethylbenzidine; KPL International). Once stopped with acid, plates were read on a spectrophotometer at $450 \mathrm{~nm}$ and data analyzed in SoftMax Pro, version 5.4 (Molecular Devices). Estimates of pharmacokinetic parameters (volume of distribution, clearance, terminal half-life) were obtained from serum concentrations using noncompartmental methods [42].

\section{NicA2 effects on blood and brain nicotine levels: Single nicotine doses}

Female Sprague Dawley rats weighing 225-250 g were purchased with jugular venous catheters in-place (Charles River Labs). Fifteen groups of 8 rats were pretreated with NicA2 through the catheter with 3 groups at each of the following NicA2 doses: $0.3125,0.625,1.25$, $5.0,10.0 \mathrm{mg} / \mathrm{kg}$. Three control groups of 8 rats each received bovine serum albumin $4 \mathrm{mg} / \mathrm{kg}$ rather than NicA2. Five min later each group received $0.03 \mathrm{mg} / \mathrm{kg}$ nicotine i.v. Six groups of rats (one group at each NicA2 dose and one control group) were then sacrificed at 1, 3 or $5 \mathrm{~min}$ following the nicotine dose. Blood and brain samples were obtained by decapitation and quenched with methanol as described above. Two additional groups were pretreated with NicA2 $20 \mathrm{mg} / \mathrm{kg}$ and studied as above but blood and brain nicotine levels measured only at 1 and 3 min based on pilot data showing blood and brain levels were undetectable at $5 \mathrm{~min}$. Blood or brain nicotine concentrations were compared by Bonferroni-corrected Welch's t-tests to accommodate heterogeneity of variance between doses or time points. Each time point or dose was considered a separate family of comparisons, such that the significance level was set at $p=0.0083$ for comparing each NicA2 dose to BSA for each time point. Serum nicotine levels were not normally distributed in two groups and brain nicotine levels were non-normal in one group. This was due to the presence of one outlier in each group, confirmed by Iterative Grubb's analysis $(p<0.01)$. These outliers were removed for the statistical analyses but were included in the figures.

\section{NicA2 effects on blood and brain nicotine levels: Multiple} nicotine doses

Male Holtzman Sprague Dawley rats weighing 340$470 \mathrm{~g}$ were anesthetized with $0.1 \mathrm{mg} / \mathrm{kg}$ fentanyl and $0.05 \mathrm{mg} / \mathrm{kg}$ dexmedetomidine i.m. and $100 \mathrm{mg} / \mathrm{kg}$ propofol i.p. and a jugular venous catheter was placed. Two groups of 10 rats received NicA2 $10 \mathrm{mg} / \mathrm{kg}$ i.v. via the jugular catheter and two groups received $10 \mathrm{mg} / \mathrm{kg}$ BSA as controls. Five min later one NicA2 and one BSA group received nicotine $0.03 \mathrm{mg} / \mathrm{kg}$ i.v. These groups were sacrificed $3 \mathrm{~min}$ after the nicotine dose, and blood and brain obtained by decapitation. The two remaining groups received nicotine $0.03 \mathrm{mg} / \mathrm{kg}$ i.v. every $10 \mathrm{~min} \times$ 5 and were sacrificed $3 \mathrm{~min}$ after the fifth nicotine dose. Blood and brain nicotine concentrations were compared between NicA2 and BSA groups using two-tailed unpaired $t$ tests with Welch's correction for unequal variances.

\section{Effect of NicA2 on nicotine discrimination}

Procedures were similar to those previously used in our laboratory [43, 44]. Four male Sprague Dawley rats weighing 550-600 g had been trained to discriminate nicotine alone $(0.4 \mathrm{mg} / \mathrm{kg}$ s.c. from saline using a 2-lever discrimination procedure). Lever pressing was reinforced under a terminal variable-interval $15 \mathrm{~s}$ schedule using $45 \mathrm{mg}$ food pellets. Discrimination was assessed twice weekly (Tues and Fri) during 2 min extinction test sessions. Discrimination was considered stable when a) $>$ $80 \%$ responding occurred on the injection-appropriate lever during two consecutive saline and nicotine test sessions, b) $>90 \%$ injection-appropriate responding occurred on six consecutive training sessions, and c) response rates (total responses/session) were stable (no trend across these four test sessions and six training sessions). When performance was stable, rats were habituated to being placed in restraint tubes for tail vein injection of NicA2 by injecting saline via tail vein 10 min prior to two or more test sessions until restraint had no effect on discrimination performance. At this point, the effect of $10 \mathrm{mg} / \mathrm{kg}$ NicA2 i.v. on the ability of $0.1 \mathrm{mg} / \mathrm{kg}$ nicotine s.c. to substitute for the $0.4 \mathrm{mg} / \mathrm{kg}$ training dose was determined. During these test sessions, PBS or NicA2 was administered i.v. $10 \mathrm{~min}$ prior to $0.1 \mathrm{mg} / \mathrm{kg}$ nicotine s.c.. The $0.1 \mathrm{mg} / \mathrm{kg}$ nicotine s.c. dose was used because it produces serum nicotine concentrations similar to those produced by the $0.03 \mathrm{mg} / \mathrm{kg}$ i.v. dose in the pharmacokinetic studies. This dose normally produces partial substitution for a $0.4 \mathrm{mg} / \mathrm{kg}$ training dose, i.e. about 50-60\% nicotine lever responding [43, 44]. The percentage of responding on the nicotine-appropriate lever (\%NLR) and overall response rate (responses/second) during the 2-min extinction test sessions served as the primary dependent measures. These measures were compared between vehicle and NicA2 administration prior to the $0.1 \mathrm{mg} / \mathrm{kg}$ nicotine substitution test sessions via paired t-test.

\section{Effect of NicA2 on nicotine or food self-administration}

Procedures were similar to those previously used in our laboratory [32]. A total of 8 male Holtzman Sprague Dawley rats weighing 310-460 g were used. Four were experimentally naïve. One was previously trained to self-administer a unit nicotine dose of $0.06 \mathrm{mg} / \mathrm{kg}$. Three had failed to self-administer anabasine in an unrelated 
pilot study. Naïve rats were implanted with jugular cannulas 1 week after arrival. One week later the rats were placed in operant conditioning chambers and allowed to acquire nicotine self-administration (NSA) using $2 \mathrm{~h}$ sessions at a $0.03 \mathrm{mg} / \mathrm{kg}$ unit nicotine dose and gradually escalating the fixed-ratio (FR) schedule to FR 3 over several weeks. Non-naïve rats were similarly trained. On average, the rats self-administered $0.51 \mathrm{mg} / \mathrm{kg} /$ day $(16.9$ \pm 4.1 SD infusions) of nicotine over the $2 \mathrm{~h}$ sessions. Rats were considered to have acquired NSA when they earned at least 8 infusions per session and responding on the active lever compared to the inactive was $>2: 1$. After at least 1 week at FR 3, if NSA was stable $(<15 \%$ variation and no trend), rats received an i.v. infusion of PBS vehicle $10 \mathrm{~min}$ before one session (Mon), followed by i.v. infusion of $20 \mathrm{mg} / \mathrm{kg}$ NicA2 $10 \mathrm{~min}$ before each of four consecutive sessions (Tues-Fri). Rats were then allowed to reacquire NSA until stable. To confirm that these rats were sensitive to changes in nicotine exposure per se, NicA2 effects were compared to extinction of NSA by substituting saline for nicotine for 4 consecutive sessions (Tues-Fri). The order of the extinction and NicA2 treatment phases was counterbalanced across subjects. Another group of 5 rats was trained to respond for sucrose pellets to examine whether NicA2 effects were selective for NSA or produced nonspecific side effects. These rats were trained under the same FR 3 schedule as the NSA group. Sessions ended after $2 \mathrm{~h}$ or when 50 pellets were earned. After responding was stable (no trend in response rate over 5 consecutive sessions), rats received an i.v. infusion of PBS vehicle $10 \mathrm{~min}$ before one session (Monday), followed by i.v. infusion of $20 \mathrm{mg} / \mathrm{kg}$ NicA2 $10 \mathrm{~min}$ before each of the following four consecutive sessions (Tues-Fri). In two rats, a higher NicA2 dose $(70 \mathrm{mg} / \mathrm{kg})$ was then tested 612 weeks later in the same manner after recovery of baseline NSA performance and allowing elimination of NicA2. Reinforcement rate (reinforcers/min) was calculated for NSA (infusions delivered) and sucrose self-administration (pellets delivered). This measure for vehicle and NicA2 test sessions was transformed to a percentage of baseline (mean of the week before NicA2 testing), which served as the primary dependent measure. Paired t-tests with a Bonferroni correction for multiple comparisons were used to compare this measure during each NicA2 session to vehicle $(p<0.012$ for four comparisons) for NSA or sucrose self-administration separately. Effects of the higher NicA2 dose were considered significant if the number of infusions during NicA2 treatment was below the range of infusions during baseline.

A separate group of four male Holtzman Sprague Dawley rats weighing 325-360 g was used in a pilot NSA study to test the longer-acting variant of the enzyme (NicA2-ABD) in rats with $23 \mathrm{~h}$ /day access to nicotine. The longer-acting enzyme allowed use of the $23 \mathrm{~h}$ nicotine access model which more closely resembles human nicotine exposure than does a $2 \mathrm{~h}$ session. Rats were trained to self-administer a unit nicotine dose of $0.03 \mathrm{mg} / \mathrm{kg}$ using the same procedures described above, except that sessions were $23 \mathrm{~h}$ in duration. Cage maintenance was done during the $1 \mathrm{~h}$ between sessions. After NSA was stable (same criteria), saline was substituted for nicotine to extinguish NSA. When the number of infusions decreased by at least $50 \%$ and there was no trend across three consecutive session, rats were allowed to reacquire stable NSA. Rats were then given a PBS vehicle infusion 10 min before one session, followed by infusion of $70 \mathrm{mg} / \mathrm{kg}$ NicA2-ABD $10 \mathrm{~min}$ prior to each of six consecutive sessions (the average number of session required for saline extinction). This dose of NicA2-ABD was used because of the variability in effect observed for the $20 \mathrm{mg} / \mathrm{kg}$ NicA2 dose during $2 \mathrm{~h}$ sessions and the higher nicotine intake occurring in the $23 \mathrm{~h}$ sessions $(1 \mathrm{mg} / \mathrm{kg} /$ day, $33.3 \pm 10.5 \mathrm{SD}$ infusions $\mathrm{v}$. $0.51 \mathrm{mg} / \mathrm{kg} /$ day for the $2 \mathrm{~h}$ sessions). Data were analyzed in the same way as the $2 \mathrm{~h}$ data $(p<0.0083$ for six comparisons).

\begin{abstract}
Abbreviations
ABD: Albumin binding domain; BChE: Butyryl cholinesterase; BSA: Bovine serum albumin; FAD: Flavin adenine dinucleotide; FR: Fixed-ratio; GABA: $\gamma^{-}$ amino-N-butyric acid; NAD: Nicotinamide adenine dinucleotide; NFDRM: Non-fat dry milk; NLR: Nicotine-appropriate lever; NSA: Nicotine selfadministration; PON: Pseudooxynicotine
\end{abstract}

\section{Acknowledgements}

We thank Theresa Harmon and Susan Walker for expert technical assistance.

\section{Funding}

This work was supported by the National Institutes of Health, National Institute on Drug Abuse (NIDA) grant R43DA044064 and by Antidote Therapeutics, Inc. NIDA funding was obtained based on the proposed study design of parts of this work but the Institute had no further role in data collection, analysis, interpretation or manuscript preparation. Several study co-authors are employees of Antidote Therapeutics Inc. and were involved in all aspects of this study.

\section{Availability of data and materials}

The datasets used and analyzed during the current study are available from the corresponding author on reasonable request.

\section{Authors' contributions}

PRP, MDR, MGL, TT, SH, and MWK participated in research design. MDR, MGL, $\Pi T, S H, Z B$ conducted experiments and made substantial contributions to the acquisition of data. PRP, MDR, MGL, $T$, SH, and MWK performed data analysis. PRP, MDR, MGL, TT, SH, and MWK contributed to the writing of the manuscript. All authors read and approved the final manuscript.

\section{Ethics approval and consent to participate}

These studies were performed in accordance with the Guide for the Care and Use of Laboratory Animals of the National Institutes of Health. Animal protocols were approved by the Minneapolis Medical Research Foundation Animal Care and Use Committee and by the Noble Life Sciences Animal Care and Use Committee. Animals were euthanized by $\mathrm{CO}_{2}$ inhalation using AAALAC approved chambers, and all efforts were made to minimize suffering. 


\section{Consent for publication}

Not applicable

\section{Competing interests}

$\mathrm{ZB}$, TT, and MWK are employees of Antidote Therapeutics, Inc.; SH is an employee of Noble Life Sciences.

\section{Publisher's Note}

Springer Nature remains neutral with regard to jurisdictional claims in published maps and institutional affiliations.

\section{Author details}

${ }^{1}$ University of Minnesota, 100 Church St. S.E, Minneapolis, MN 55455, USA. ${ }^{2}$ Minneapolis Medical Research Foundation, 701 Park Ave, Minneapolis, MN 55415, USA. ${ }^{3}$ Antidote Therapeutics Inc, 708 Quince Orchard Road, Suite 250-C, Gaithersburg, MD 20878, USA. ${ }^{4}$ Noble Life Sciences, PO Box 242, Woodbine, MD 21797, USA.

Received: 30 April 2018 Accepted: 12 July 2018

Published online: 24 July 2018

\section{References}

1. Anon. The health consequences of smoking: Nicotine addiction, a report of the Surgeon General. Atlanta: U.S. Department of Health and Human Services, Centers for Disease Control and Prevention, Office on Smoking and Health; 1988.

2. Prochaska JJ, Benowitz NL. The past, present, and future of nicotine addiction therapy. Annu Rev Med. 2016;67:467-86.

3. Fiore M. Treating tobacco use and dependence. Clinical practice guideline, 2008 update: U.S. Department of Health and Human Services: Public Health Service; 2008.

4. Pentel PR, LeSage MG. New directions in nicotine vaccine design and use. Adv Pharmacol. 2014;69:553-80.

5. Hartmann-Boyce J, Cahill K, Hatsukami D, Cornuz J. Nicotine vaccines for smoking cessation. Cochrane Database Syst Rev. 2012:8:CD007072.

6. Cornuz J, Zwahlen S, Jungi WF, Osterwalder J, Klingler K, van Melle G, Bangala Y, Guessous I, Muller P, Willers J, et al. A vaccine against nicotine for smoking cessation: a randomized controlled trial. PLoS One. 2008;3(6):e2547.

7. Hatsukami DK, Jorenby DE, Gonzales D, Rigotti NA, Glover ED, Oncken CA Tashkin DP, Reus VI, Akhavain RC, Fahim RE, et al. Immunogenicity and smoking-cessation outcomes for a novel nicotine immunotherapeutic. Clin Pharmacol Ther. 2011;89(3):392-9.

8. Xue $\mathrm{S}, \mathrm{Schlosburg} J \mathrm{E}$, Janda KD. A new strategy for smoking cessation: characterization of a bacterial enzyme for the degradation of nicotine. J Am Chem Soc. 2015;137(32):10136-9.

9. Xue S, Kallupi M, Zhou B, Smith LC, Miranda PO, George O, Janda KD. An enzymatic advance in nicotine cessation therapy. Chem Commun (Camb). 2018:54(14):1686-9.

10. Hylin JW. Microbial degradation of nicotine. I. Morphology and physiology of Achromobacter nicotinophagum n. sp. J Bacteriol. 1958;76(1):36-40.

11. Brandsch R. Microbiology and biochemistry of nicotine degradation. Appl Microbiol Biotechnol. 2006;69(5):493-8.

12. Tang H, Wang L, Wang W, Yu H, Zhang K, Yao Y, Xu P. Systematic unraveling of the unsolved pathway of nicotine degradation in Pseudomonas. PLoS Genet. 2013;9(10):e1003923.

13. Wang SN, Xu P, Tang HZ, Meng J, Liu XL, Qing C. "Green" route to 6hydroxy-3-succinoyl-pyridine from (S)-nicotine of tobacco waste by whole cells of a Pseudomonas sp. Environ Sci Technol. 2005;39(17):6877-80.

14. Yu H, Tang H, Wang L, Yao Y, Wu G, Xu P. Complete genome sequence of the nicotine-degrading Pseudomonas putida strain S16. J Bacteriol. 2011; 193(19):5541-2.

15. Hukkanen J, Jacob P 3rd, Benowitz NL. Metabolism and disposition kinetics of nicotine. Pharmacol Rev. 2005;57(1):79-115.

16. Bardo MT, Bevins RA, Klebaur JE, Crooks PA, Dwoskin LP. (-)-Nornicotine partially substitutes for (+)-amphetamine in a drug discrimination paradigm in rats. Pharmacol Biochem Behav. 1997;58(4):1083-7.

17. Hoffman AC, Evans SE. Abuse potential of non-nicotine tobacco smoke components: acetaldehyde, nornicotine, cotinine, and anabasine. Nicotine Tob Res. 2013;15(3):622-32.
18. Tararina MA, Janda KD, Allen KN. Structural analysis provides mechanistic insight into nicotine oxidoreductase from Pseudomonas putida. Biochemistry. 2016;55(48):6595-8.

19. Harris AC, Pentel PR, Lesage MG. Prevalence, magnitude, and correlates of an extinction burst in drug-seeking behavior in rats trained to selfadminister nicotine during unlimited access ( $23 \mathrm{~h} /$ day) sessions. Psychopharmacology. 2007;194(3):395-402.

20. Nasser AF, Fudala PJ, Zheng B, Liu Y, Heidbreder C. A randomized, doubleblind, placebo-controlled trial of RBP-8000 in cocaine abusers: pharmacokinetic profile of rbp-8000 and cocaine and effects of RBP-8000 on cocaine-induced physiological effects. J Addict Dis. 2014;33(4):289-302.

21. Shram MJ, Cohen-Barak O, Chakraborty B, Bassan M, Schoedel KA, Hallak H, Eyal E, Weiss S, Gilgun-Serki Y, Sellers EM, et al. Assessment of pharmacokinetic and Pharmacodynamic interactions between albumin-fused mutated Butyrylcholinesterase and intravenously administered cocaine in recreational cocaine users. J Clin Psychopharmacol. 2015:35(4):396-405.

22. Smethells JR, Swalve N, Brimijoin S, Gao Y, Parks RJ, Greer A, Carroll ME. Long-term blockade of cocaine self-administration and locomotor activation in rats by an adenoviral vector-delivered cocaine hydrolase. J Pharmacol Exp Ther. 2016;357(2):375-81.

23. Lofwall MR, Nuzzo PA, Campbell C, Walsh SL. Aripiprazole effects on selfadministration and pharmacodynamics of intravenous cocaine and cigarette smoking in humans. Exp Clin Psychopharmacol. 2014;22(3):238-47.

24. Amundsen EJ, Reid MJ. Self-reports of consumption of amphetamines, cocaine and heroin in a survey among marginalized drug users. Sci Total Environ. 2014;487:740-5.

25. Benowitz NL, Perez-Stable EJ, Herrera B, Jacob P 3rd. Slower metabolism and reduced intake of nicotine from cigarette smoking in ChineseAmericans. J Natl Cancer Inst. 2002;94(2):108-15.

26. Gao Y, LaFleur D, Shah R, Zhao Q, Singh M, Brimijoin S. An albuminbutyrylcholinesterase for cocaine toxicity and addiction: catalytic and pharmacokinetic properties. Chem Biol Interact. 2008;175(1-3):83-7.

27. LeSage MG, Keyler DE, Shoeman D, Raphael D, Collins G, Pentel PR. Continuous nicotine infusion reduces nicotine self-administration in rats with 23-h/day access to nicotine. Pharmacol Biochem Behav. 2002; 72(1-2):279-89.

28. Keyler DE, Roiko SA, Benlhabib E, LeSage MG, St Peter JV, Stewart S, Fuller S, Le CT, Pentel PR. Monoclonal nicotine-specific antibodies reduce nicotine distribution to brain in rats: dose- and affinity-response relationships. Drug Metab Dispos. 2005;33(7):1056-61.

29. Pentel PR, Malin DH, Ennifar S, Hieda Y, Keyler DE, Lake JR, Milstein JR, Basham LE, Coy RT, Moon JW, et al. A nicotine conjugate vaccine reduces nicotine distribution to brain and attenuates its behavioral and cardiovascular effects in rats. Pharmacol Biochem Behav. 2000;65(1):191-8.

30. Chaudhury C, Mehnaz S, Robinson JM, Hayton WL, Pearl DK, Roopenian DC, Anderson CL. The major histocompatibility complex-related fC receptor for lgG (FcRn) binds albumin and prolongs its lifespan. J Exp Med. 2003;197(3):315-22.

31. Harris AC, Tally L, Schmidt CE, Muelken P, Stepanov I, Saha S, Vogel RI, LeSage MG. Animal models to assess the abuse liability of tobacco products: effects of smokeless tobacco extracts on intracranial selfstimulation. Drug Alcohol Depend. 2015;147:60-7.

32. LeSage MG, Burroughs D, Muelken P, Harris AC. Self-Administration of Smokeless Tobacco Products in rats. Tob Regul Sci. 2016;2(4):329-42.

33. Gellner CA, Belluzzi JD, Leslie FM. Self-administration of nicotine and cigarette smoke extract in adolescent and adult rats. Neuropharmacology. 2016;109:247-53.

34. Seeman Jl, Dixon M, Haussmann HJ. Acetaldehyde in mainstream tobacco smoke: formation and occurrence in smoke and bioavailability in the smoker. Chem Res Toxicol. 2002;15(11):1331-50.

35. Smith TT, Rupprecht LE, Cwalina SN, Onimus MJ, Murphy SE, Donny EC, Sved AF. Effects of monoamine oxidase inhibition on the reinforcing properties of low-dose nicotine. Neuropsychopharmacology. 2016;41(9): 2335-43.

36. Donny EC, Denlinger RL, Tidey JW, Koopmeiners JS, Benowitz NL, Vandrey RG, al'Absi M, Carmella SG, Cinciripini PM, Dermody SS, et al. Randomized trial of reduced-nicotine standards for cigarettes. N Engl J Med. 2015; 373(14):1340-9

37. Gill SC, von Hippel PH. Calculation of protein extinction coefficients from amino acid sequence data. Anal Biochem. 1989;182(2):319-26. 
38. Zhou M, Panchuk-Voloshina N. A one-step fluorometric method for the continuous measurement of monoamine oxidase activity. Anal Biochem. 1997;253(2):169-74.

39. Jonsson A, Dogan J, Herne N, Abrahmsen L, Nygren PA. Engineering of a femtomolar affinity binding protein to human serum albumin. Protein Eng Des Sel. 2008;21(8):515-27.

40. Jacob P, Wilson M, Benowitz NL. Improved gas chromatographic method for the determination of nicotine and cotinine in biologic fluids. J Chromatogr. 1981;222:61-70.

41. Hieda Y, Keyler DE, Vandevoort JT, Kane JK, Ross CA, Raphael DE, Niedbalas RS, Pentel PR. Active immunization alters the plasma nicotine concentration in rats. J Pharmacol Exp Ther. 1997;283(3):1076-81.

42. Zhang Y, Huo M, Zhou J, Xie S. PKSolver: an add-in program for pharmacokinetic and pharmacodynamic data analysis in Microsoft excel. Comput Methods Prog Biomed. 2010:99(3):306-14.

43. LeSage MG, Shelley D, Pravetoni M, Pentel PR. Enhanced attenuation of nicotine discrimination in rats by combining nicotine-specific antibodies with a nicotinic receptor antagonist. Pharmacol Biochem Behav. 2012; 102(1):157-62

44. LeSage MG, Shelley D, Ross JT, Carroll Fl, Corrigall WA. Effects of the nicotinic receptor partial agonists varenicline and cytisine on the discriminative stimulus effects of nicotine in rats. Pharmacol Biochem Behav. 2009;91(3):461-7.

Ready to submit your research? Choose BMC and benefit from:

- fast, convenient online submission

- thorough peer review by experienced researchers in your field

- rapid publication on acceptance

- support for research data, including large and complex data types

- gold Open Access which fosters wider collaboration and increased citations

- maximum visibility for your research: over $100 \mathrm{M}$ website views per year

At $\mathrm{BMC}$, research is always in progress.

Learn more biomedcentral.com/submissions 